\title{
STAGE AND IMMUNOHISTOCHEMISTRY OF EIGHT THOUSAND CASES OF BREAST CANCER TREATED IN A BRAZILIAN REFERENCE CENTRE
}

Luiz Henrique Gebrim¹, André Mattar¹, Jorge Yoshinori Shida1, Roberto Hegg1

1Women's Reference Center, Hospital Pérola Byington - São Paulo (SP), Brazil.

Objetives: Breast cancer affects about 59,000 Brazilian women annually according to data from INCA 2018 (National Cancer Institute) and 14,388 deaths are related to the disease. There are few data about the stage of the disease in our Country. Most of the cases are confirmed in late stages mainly due to the delay in diagnosis and time to initiate the treatment. Pérola Byington Hospital is one of the most important cancer centers specialized breast cancer treatment in Brazil and we are responsible for treating about a thousand new cases of breast cancer annually. In 2005 a model of visits based on quick diagnosis and treatment was implemented named CARE (Centro de Alta Resolutividade - High Resolutivity Center) based on a model of One Stop Clinic. This approach can lead to a downstage of breast cancer stage once the diagnosis and the treatment is substantially quicker. In this study we have evaluated the stage and immunohistochemistry (IHC) subtype of the patients treated in our institution. Methodology: In 2016 we have created a web-based system that helped us to input information about the breast câncer cases treated in Pérola Byington Hospital. This program helped us to better understand our cases of breast cancer regarding the initial stage and the subtype of breast cancer based on IHQ. The main idea was to analyze our data and compare it with the national data and the international literature. Results: We have reviewed 8,420 cases of breast cancer treated in our Hospital between 2011 and 2017. We were able to access the clinical stage in 8,096 cases. The distribution was $4.9 \%$ of stage $0,22.9 \%$ of stage I, $39.2 \%$ of stage II, $30 \%$ of stage III and $3.1 \%$ of stage IV at the time of diagnosis. The data regarding the immunohistochemistry subtype was able to be recovered in 7,934 patients. Most of our cases were luminal (A or B) - 56.4\%. There was $10.3 \%$ of both Her2 and hormonal receptor positive and $7.9 \%$ of only Her2 positive. The prevalence in our patients of triple negative disease was $25.4 \%$ much higher than the literature (around 15\%). We have also looked at the Her2 doubtful (Her2 2+) where usually you need a specific test to define it (CISH or FISH test). There were 1,450 cases in this condition and in $28.6 \%$ of them the test was not made. Among the cases that made the test we found that $74.7 \%$ resulted in CISH negative, $14.1 \%$ positive and $11.2 \%$ were still inconclusive. The Ministry of Health forces all the Her2 positive in the IHC (3/3) to be tested with a FISH or a CISH test. We found 616 cases of Her2 positive in the IHQ test and negative when CISH was made (7.2\%). Conclusions: In our analysis we have found that our institution has more cases of triple negative disease than reported in the literature. Based on national statistics we had substantially fewer cases of advanced disease (only $30 \%$ of stage III compared to $60 \%$ of INCA report). Probably these findings are related to the quick diagnosis and immediate treatment (surgical or neoadjuvant). There was $17.4 \% \%$ of cases found to have Her2 uncertain and most of them were tested and became a Her2 negative when CISH was performed (74.7\%). We have to look carefully to those patients with Her2 positive in IHC but negative when CISH was done specially because they do not receive trastuzumab according to Brazilian Ministry of Health guidelines a a further study will be made to evaluate that. 